Theme: Parenting/parenthood.

Keywords: Kangaroo mother care, neonatal intensive care, parents, stress.

Introduction: Early maternal contact can protect the infants' brain from harmful effects of stress while deprivation increases the stress level and leads to increased sensitivity to stress.

Aim: To evaluate the effects of continuous skin-to-skin contact (SSC) after preterm birth on stress.

Methods: Late preterm infants from two neonatal care units were randomized to either SSC or standard care. Salivary cortisol was measured in response to a nappy change at one month, and again at four months in response to a still-face procedure.

Results: Infants randomized to SSC had a significantly lower salivary cortisol reactivity at one month and there was a correlation between the mothers' and the preterm infants' salivary cortisol levels at four months.

Conclusions: The results show that close parental contact and human touch have a buffering effect on the infant's stress reactivity and stimulate a more rapid development of regularity.

\title{
OC10 - Inter-rater agreement of the Paediatric Early Warning Score tools used in the central Denmark region
}

\author{
Claus Sixtus Jensen (Denmark) ${ }^{1,2}$; Hanne Aagaard (Denmark); \\ Hanne Vebert Olesen (Denmark) ${ }^{3} ;$ Hans Kirkegaard (Denmark) ${ }^{1}$
}

\author{
${ }^{1}$ Research Center for Emergency Medicine, Aarhus University Hospital; ${ }^{2}$ Aarhus \\ University; ${ }^{3}$ Department of Paediatrics, Aarhus University Hospital
}

Theme: Patient safety

Keywords: Inter-rater agreement, Paediatric Early Warning Score, PEWS, reliability.

Background: Paediatric Early Warning Score (PEWS) tools can assist healthcare providers in the rapid detection and recognition of changes in patient condition. In the central Denmark region two different PEWS tools tested in large-scale RCT study. However, data from PEWS instruments are only as reliable and accurate as the caregiver who obtains and documents the parameters.

Aim: The purpose was to evaluate the inter-rater agreement among nurses using the PEWS systems.

Design: The study was conducted in five paediatrics departments. Inter-observer reliability was investigated through simultaneous blinded PEWS assessment on the same patients by two nurses.

Fleiss' kappa was utilized to determine the level of agreement among the raters.

Conclusion: With a paucity of published reliability testing studies, this research attempts to address identified research gaps and will thus inform nursing practice. 